\title{
A compound heterozygous mutation of the alkaline phosphatase ALPL gene causes hypophosphatasia in a Han Chinese family
}

\author{
HUAJIE HUANG ${ }^{1,2^{*}}$, JIAN WANG ${ }^{3 *}$, YINGYIN LIANG ${ }^{4}$, XIAOFENG WEI ${ }^{1}$, DAN GUO ${ }^{1}$, \\ HENGBIAO SUN ${ }^{5}$, XUELIAN ZHANG $^{1}$, XIANGMIN XU $^{1,6}$ and FU XIONG ${ }^{1,6}$ \\ ${ }^{1}$ Department of Medical Genetics, School of Basic Medical Sciences, Southern Medical University, Guangzhou, \\ Guangdong 510515; ${ }^{2}$ Medical Genetic Centre, Guangdong Women and Children Hospital, Guangzhou, Guangdong 511400; \\ ${ }^{3}$ Department of Orthopedic Surgery, Southern Medical University, Nanfang Hospital, Guangzhou, Guangdong 510515; \\ ${ }^{4}$ Department of Neurology, First Affiliated Hospital, Sun Yat-sen University, Guangzhou, Guangdong 510080; \\ ${ }^{5}$ Department of Clinical Laboratory, The Third Affiliated Hospital of Southern Medical University, \\ Guangzhou, Guangdong 510630; ${ }^{6}$ Guangdong Provincial Key Laboratory of Single Cell \\ Technology and Application, Guangzhou, Guangdong 510515, P.R. China
}

Received June 26, 2019; Accepted April 7, 2020

DOI: $10.3892 / \mathrm{etm} .2020 .9281$

\begin{abstract}
Hypophosphatasia (HPP) is a rare hereditary systemic disease that is characterized by defective bone and/or dental mineralization, and is caused by mutations in the alkaline phosphatase gene (ALPL). The present study investigated the ALPL mutation in a Chinese Han family with HPP and studied the pathogenesis of the mutations of the ALPL gene. DNA was extracted from peripheral venous blood of the family members. Sanger sequencing was used to screen the mutations. Associations between pathogenesis for both mutations were analyzed by bioinformatics, subcellular localization, measurement of enzyme activity and western blotting. Sanger sequencing revealed the compound heterozygous mutations c.203C $>\mathrm{T}$ (p.T68M) and c.571G $>\mathrm{A}$ (p.E191K). The mutations were located at exon 4 and 6 of the ALPL gene and were predicted by Polyphen- 2 analysis to be harmful. Protein analysis indicated a decrease in mature protein production and lower enzyme activity in 293T cells transfected with plasmids carrying the mutations. The ALPL gene was cloned into the pcDNA3.1(+) vector and mutant plasmids ALPL-pT68M and ALPL-pE191K were constructed. Immunofluorescence observed in cells transfected with the ALPL-pE191K mutant plasmid was mainly located in the cell
\end{abstract}

Correspondence to: Professor $\mathrm{Fu}$ Xiong, Department of Medical Genetics, School of Basic Medical Sciences, Southern Medical University, 1838 Guangzhou Avenue, Guangzhou, Guangdong 510515, P.R. China

E-mail: xiongfu@smu.edu.cn

${ }^{*}$ Contributed equally

Key words: alkaline phosphatase, compound heterozygous, hypophosphatasia membrane. However, staining in the cytoplasm was increased compared with the wild type, and almost no fluorescence was identified in 293T cells transfected with the ALPL-pT68M mutant plasmid. The present findings demonstrated that the compound heterozygous c. $571 \mathrm{G}>\mathrm{A}$ and c.203C $>\mathrm{T}$ mutations may contribute to childhood HPP by resulting in mislocalization, decreased protein expression and loss of enzyme activity in a Han Chinese family. The results of the current study may provide insights into the potential molecular mechanism of HPP.

\section{Introduction}

Hypophosphatasia (HPP) is a rare skeletal systemic disease that was first described by Rathbun in 1948 (1). HPP can be inherited in an autosomal recessive or autosomal dominant manner (1). A total of 1 in 25 individuals carry the alkaline phosphatase gene (ALPL) founder mutation and $\sim 1$ in 2,500 newborns suffer from lethal HPP among Mennonites in Canada (2,3). A previous study of ALPL mutation analysis predicted that the incidence of severe and moderately severe HPP in Europe was 1/300,000 and 1/6,370, respectively, in 2011 (4).

HPP appears as a defective mineralization of the bone or teeth with low serum activity and bone alkaline phosphatase (5). Clinical features are varied and range from the prenatal lethal form without mineralized bone to adult HPP with pathological fractures of the lower limbs in adulthood (6,7). A total of six clinical forms, depending on age at diagnosis and severity of symptoms, are distinguished (8). Severe perinatal HPP is characterized by respiratory failure and hypercalcemia $(8,9)$. Benign perinatal HPP displays prenatal skeletal anomalies $(8,10)$. Clinical symptoms of infantile HPP appear between birth and six months of age and include craniosynostosis and rickets without elevated serum alkaline phosphatase activity $(6,11,12)$. Features of childhood HPP mainly comprise low bone mineral density with unexplained fractures and early 
loss of primary teeth (7). Patients with adult HPP present with stress fractures and pseudofractures of the lower limbs in middle age, occasionally accompanied by premature loss of permanent teeth (13-15). Odontohypophosphatasia presents with premature exfoliation of primary teeth or severe dental caries $(6,8)$.

Alkaline phosphatases (ALP), also known as glycoprotein enzymes, catalyze the hydrolysis of phosphoesters to release inorganic phosphate (16). There are four different ALP isoenzymes, three tissue-specific ALPs (placenta, intestine and germ cell) and one tissue-non-specific ALP (TNAP or TNSALP) (17-20). TNAP, which is encoded by the ALPL gene, is not only highly expressed in bone, liver, kidney and intestinal mucosa, but also in brain, fibroblasts and endothelium $(6,21)$.

The current study presented with a young Chinese male with HPP caused by a compound heterozygous mutation in ALPL inherited from his parents. The results of the present study on the function and potential pathogenic mechanism of HPP suggested that the compound heterozygous mutations led to diminished expression of ALPL, which ultimately caused HPP. The present research may provide a reference for future studies of the potential pathogenical mechanisms by which mutations in ALPL cause HPP.

\section{Materials and methods}

Patients. The patient and his family members were recruited in Nanfang Hospital. The proband (III-1), aged six, was referred to the Surgery of Joints and Osteopathy at Nanfang Hospital, China for consultation associated with skeletal pain and premature loss of primary teeth between March and April 2016. Physical and imaging examinations of the proband were performed by an experienced orthopedic surgeon. Osteoporosis was investigated by X-ray imaging. A total of 200 healthy controls were randomly recruited from the Department of Medical Genetics, School of Basic Medical Sciences, Southern Medical University, China between June and September 2016. The criteria for inclusion were matched ethnic origin, age range between 20-50 years and healthy individuals without major diseases.

Mutation screening. Genomic DNA was isolated from the peripheral blood of the proband and his family members using standard phenol/chloroform extraction. A total of $2 \mathrm{ml}$ EDTA-anticoagulated whole blood was added to a $15 \mathrm{ml}$ tube with $10 \mathrm{ml}$ water for solvation of erythrocytes and centrifuged for $10 \mathrm{~min}$ at 2,580 $\mathrm{x} \mathrm{g}$ at room temperature. The supernatant was discarded and the aforementioned step was repeated. A total of $500 \mu \mathrm{l}$ sodium chloride-Tris-EDTA buffer $(0.1 \mathrm{M} \mathrm{NaCl}, 0.01 \mathrm{M}$ Tris-HCl pH 8.0, 0.001 M $\mathrm{Na}_{2}$ EDTA $\mathrm{pH} 8.0$ ), $50 \mu \mathrm{l} 10 \%$ SDS and $5 \mu \mathrm{l} 10 \mathrm{mg} / \mathrm{ml}$ proteinase $\mathrm{K}$ were added to the tube and the tube was shaken overnight at $55^{\circ} \mathrm{C}$. The lysate was transferred to a $1.5 \mathrm{ml}$ Eppendorf tube. A total of $600 \mu \mathrm{l}$ saturated phenol was added to the tube, vortexed vigorously and centrifuged for $5 \mathrm{~min}$ at $13,524 \mathrm{x} \mathrm{g}$ at room temperature. The aqueous phase was transferred to a new $1.5 \mathrm{ml}$ tube and $300 \mu \mathrm{l}$ saturated phenol and $300 \mu \mathrm{l}$ buffer-chloroform/isoamylol was added. The tube was vigorously vortexed, centrifuged for $5 \mathrm{~min}$ at $13,524 \mathrm{x} \mathrm{g}$ at room temperature and the aqueous phase was transferred to another new tube followed by addition of $600 \mu \mathrm{l}$ buffer-chloroform//isoamylol. The tube was centrifuged for $2 \mathrm{~min}$ at 13,524 $\mathrm{x} g$ at room temperature and the supernatant was transferred to a new tube. Subsequently, $60 \mu 13 \mathrm{M}$ sodium acetate and $1.2 \mathrm{ml}$ ethanol was added to the tube, and the tube was stored at $-20^{\circ} \mathrm{C}$ for $20 \mathrm{~min}$. The tube was then centrifuged for $12 \mathrm{~min}$ at $13,524 \mathrm{x} \mathrm{g}$, left to precipitate and washed with $75 \%$ ethanol twice. The precipitate was dried and resuspended in $50 \mu \mathrm{l}$ TE buffer (0.1 M Tris- $\mathrm{HCl}$ pH8.0 and 0.01 M Na${ }_{2}$ EDTA). All exons of ALPL were amplified by PCR using Taq DNA polymerase (Beijing Dingguo Changsheng Biotechnology Co., Ltd.). Agarose gel (1.2\%) electrophoresis and Sanger sequencing were used to analyze the PCR products. Two significant mutations within exons 4 and 6 were identified. The following primer pairs were used for the PCR: ALPL exon 4 forward, 5'-CTTACCCCGCCAAGTAACTG-3' and reverse, 5'-ACA GACCTGAACTGGGCTTG-3' and ALPL exon 6 forward, 5'-ACACCCCGATCTGTGGATAA-3' and reverse, 5'-CAA CCGCAAATCCCCTAAT-3'. The following thermocycling conditions were used for the PCR: Initial denaturation for $5 \mathrm{~min}$ at $95^{\circ} \mathrm{C}$; followed by 35 cycles of denaturation $(30 \mathrm{sec}$; $95^{\circ} \mathrm{C}$ ), annealing $\left(30 \mathrm{sec} ; 60^{\circ} \mathrm{C}\right)$, extension $(45 \mathrm{sec}$ for exon 4; $30 \mathrm{sec}$ for exon $6 ; 72^{\circ} \mathrm{C}$ ), and a final extension for $10 \mathrm{~min}$ at $72^{\circ} \mathrm{C}$.

Bioinformatics. The secondary and three-dimensional structures of wild-type (WT) and mutant ALPL proteins were predicted using the Self-Optimized Prediction method with the Alignment (SOPMA) method (22), Iterative Threading ASSEmbly Refinement (I-TASSER; http://zhanglab.ccmb. med.umich.edu/I-TASSER/) and SWISS-MODEL (based on P05816) (https://swissmodel.expasy.org/), respectively. PolyPhen-2 (http://genetics.bwh.harvard.edu/pph2/), SIFT (http://sift.jcvi.org/) and Mutation Taster (http://www. mutationtaster.org/) were used to predict the harmfulness of mutations and the University of California, Santa Cruz (UCSC) Genome Browser (http://genome.ucsc.edu) was used to analyze the conservation of ALPL across species.

Cellular localization. The coding sequence of the ALPL gene was cloned into the BamHI and EcoRI sites of the pcDNA3.1(+) vector (Invitrogen; Thermo Fisher Scientific, Inc.). The plasmid was used as a template for generating the mutant plasmids ALPL-pT68M and ALPL-pE191K. The following primer pairs were used for the PCR: c.203C $>\mathrm{T}$ forward, 5'-GTGTCTCCACAGTGATGGCTGCCCGCATCC T-3' and reverse, 5'-AGGATGCGGGCAGCCATCACTGTG GAGACAC-3' and c.571G>A forward, 5'-TGGTACTCAGAC AACAAGATGCCCCCTGAGG-3' and reverse, 5'-CCTCAG GGGGCATCTTGTTGTCTGAGTACCA-3'. The following thermocycling conditions were used for the PCR: Initial denaturation for $5 \mathrm{~min}$ at $95^{\circ} \mathrm{C}$; followed by 20 cycles of denaturation $\left(10 \mathrm{sec} ; 98^{\circ} \mathrm{C}\right)$, annealing $\left(15 \mathrm{sec} ; 68^{\circ} \mathrm{C}\right)$, extension $\left(7 \mathrm{~min} ; 72^{\circ} \mathrm{C}\right)$; and a final extension for $10 \mathrm{~min}$ at $72^{\circ} \mathrm{C}$. 293 T cells (Guangzhou Saiku Biotechnlogy Co., Ltd.) were grown in DMEM (Invitrogen; Thermo Fisher Scientific, Inc.) supplemented with 10\% FBS (Gibco; Thermo Fisher Scientific, Inc.) at $37^{\circ} \mathrm{C}$ and transiently transfected with recombinant 
constructs $(3 \mu \mathrm{g})$ using Lipofectamine ${ }^{\mathrm{TM}} 2000$ (Invitrogen; Thermo Fisher Scientific, Inc.) for $48 \mathrm{~h}$.

Immunostaining. Following $48 \mathrm{~h}$ of transfection, cells were washed with PBS (pH 7.4) three times, fixed with ice-cold methanol for $10 \mathrm{~min}$, and dried and washed with PBS. Cells were then permeabilized for $15 \mathrm{~min}$ with PBS-Tween-20 (0.05\%) and washed with PBS three times. Subsequently, cells were blocked with 3\% BSA (Beyotime Institute of Biotechnology) and incubated with mouse anti-TNAP (1:50; cat. no. sc21708; Santa Cruz Biotechnology, Inc.) at $4^{\circ} \mathrm{C}$ for $16 \mathrm{~h}$ and stained with FITC-conjugated secondary antibodies (1:200; cat. no. F2012; Sigma-Aldrich; Merck KGaA) at room temperature for $2 \mathrm{~h}$. Cell nuclei were stained with DAPI (Sigma-Aldrich; Merck KGaA) for $10 \mathrm{~min}$ at room temperature. Fluorescence microscopy (LSM 880; Carl Zeiss AG) was used to visualize the fluorescence signal of transfected cells at x100, magnification.

ALPL expression in $293 T$ cells. To determine the expression of WT and mutant ALPL, recombinant constructs were transfected into $293 \mathrm{~T}$ cells using Lipofectamine ${ }^{\mathrm{TM}} 2000$ (Invitrogen; Thermo Fisher Scientific, Inc.). At 24 or $48 \mathrm{~h}$ after transfection, cells were harvested for protein extraction with radioimmunoprecipitation assay buffer (Cell Signaling Technology, Inc.) supplemented with protease inhibitor cocktail (Sigma-Aldrich; Merck KgaA). The lysates were quantified using a bicinchoninic acid assay (Thermo Fisher Scientific, Inc.) and then boiled with protein loading buffer (Beyotime Insititute of Biotechnology). A total of $20 \mu \mathrm{g}$ protein/lane was separated by SDS-PAGE with 5\% stacking gel and $10 \%$ separation gels. Subsequently, proteins were transferred to polyvinylidene fluoride membranes (EMD Millipore). The membranes were blocked with 5\% nonfat milk at room temperature for $1 \mathrm{~h}$ and then incubated with mouse anti-TNAP (1:100; cat. no. sc21708; Santa Cruz Biotechnology, Inc.) overnight at $4^{\circ} \mathrm{C}$ and incubated with goat-anti-mouse immunoglobulin G-horseradish peroxidase (HRP; 1:4,000; cat. no. RM3001; Beijing Ray Antibody Biotech) at room temperature for $1 \mathrm{~h}$. Signal detection was performed using the Immobilon Western Chemiluminescent HRP substrate (EMD Millipore; Merck KGaA).

TNAP relative enzyme activity test. $293 \mathrm{~T}$ cells were transfected with recombinant constructs and an alkaline phosphatase detection kit (cat. no. P0321; Beyotime Institute of Biotechnology) was used to investigate the relative TNAP enzyme activity of WT and mutant ALPL after transfection for 24 or $48 \mathrm{~h}$.

Statistical analysis. Statistical analysis was performed using GraphPad Prism 5 (GraphPad Software Inc.). Statistical differences among the four groups were determined using one-way ANOVA followed by Bonferroni's correction. Data are presented as the mean \pm SEM and the experiments were repeated three times. ${ }^{\text { }} \mathrm{P}<0.05$ was considered to indicate a statistically significant difference.

\section{Results}

Clinical features. The results of the physical examination indicated that the proband exhibited cerebral abnormalities,

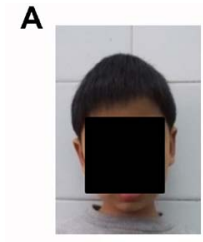

D

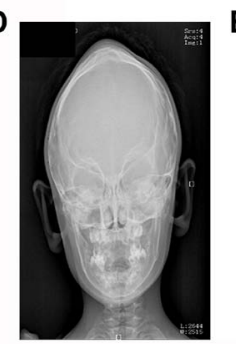

B

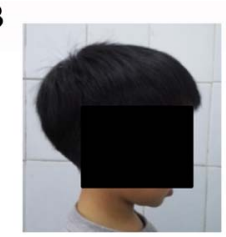

E

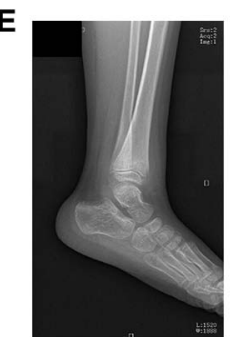

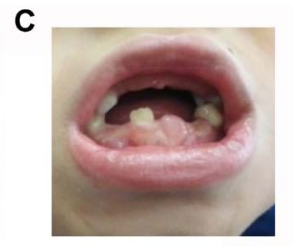

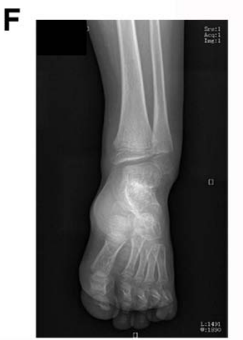

Figure 1. Clinical manifestations of the proband. The proband presented with (A) scaphocephaly and (B) a long, narrow head. (C) The primary teeth were almost lost when he was 5 years old. (D) Head CT image results revealed that the anteroposterior diameter of the cranial cavity was larger and the biparietal diameter was shorter than normal when the proband was 1 year old (E) X-ray image of the proband's left ankle at 5 years (lateral film). (F) X-ray image of the proband's left ankle (anteroposterior film).

premature loss of primary teeth (Fig. 1A-C), and mild pigeon breast. Head CT scan results of the proband when he was 1 year of age revealed that the anteroposterior diameter of the cranial cavity was larger and biparietal diameter was shorter than normal. In addition, the patient's forehead was extremely prominent, while the sagittal suture was closed and the coronal seam and lambdoidal suture were present, which indicated that scaphocephaly resulted from premature fusion of cranial sutures (Fig. 1D). X-ray findings of the left ankle indicated decreased bone density and loosened bone trabeculae. In addition, the distal tibiofibula was slightly enlarged so that it looked like a brush and the epiphysis of the distal fibula was less regular with soft tissue around it, causing left ankle joint swelling (Fig. 1E and F). Serum alkaline phosphatase activity of the proband (III-3) was below the normal reference range, while that of his mother (II-4) was near the threshold, and that of his father (II-3) was normal (Table I).

Identification of ALPL mutations. Genetic analysis of the ALPL gene from all family members was performed, and two variants that were not indicated in the 200 control samples were identified. The c.571G $>$ A mutation [GenBank accession no. NM_000478.5, p.Glu191Lys; Human Gene Mutation Database (HGMD) accession no. CM920019] within exon 6 of ALPL is a common pathogenic mutation which has been previously identified (Fig. 2) (23). The c.203C $>$ T mutation (GenBank accession no. NM_000478.5, p.Thr68Met) within exon 4 was revealed to be an allele associated with severe childhood HPP and is considered to be pathogenic (HGMD accession no. CM012046) (24). The frequency of ALPL 571A allele (ClinVar accession no. rs121918007) is 0.00247 in Genome Aggregation Database (gnomAD; http://gnomad.broadinstitute.org/) and 0.00258 in Exome Aggregation Consortium (ExAC; https://gnomad.broadinstitute.org/) However, the frequency of ALPL 203T allele (chr1:21887611) was not able to be indicated in ExAC or gnomAD. Sanger sequencing indicated that the proband presented both heterozygous mutations 
Table I. Clinical data and laboratory parameters of the HPP proband (III-1) and his parents (II-3 and II-4).

\begin{tabular}{lcccccccc}
\hline & & & \multicolumn{3}{c}{ Serum } & & \multicolumn{2}{c}{ Urine } \\
\cline { 7 - 8 } Name & Sex & Age & Ca (mmol/l) & P (mmol/l) & ALP (IU/l) & & 24 h Ca (mmol) & 24 h P (mmol) \\
\hline II-3 & M & 39 & 2.34 & 0.99 & 45 & & 5.3 & 18.43 \\
II-4 & F & 34 & 2.34 & 1.16 & 29 & & 2.2 & 18.01 \\
III-1 & M & 6 & 2.35 & 1.65 & 41 & & 1.55 & 6.58 \\
\hline
\end{tabular}

Reference ranges: Serum calcium, 2.1-2.65 mmol/l; serum phosphorus, 0.8-1.5 mmol/1; ALP for adults, 29-111 IU/l; ALP for boys aged six, 110-341 IU/l; 24 h urine calcium 0-7.5 mmol; 24 h urine phosphorus 13-42 mmol. HPP, hypophosphatasia; Ca, calcium; P, phosphorus; ALP, alkaline phosphatase; M, male; F, female.
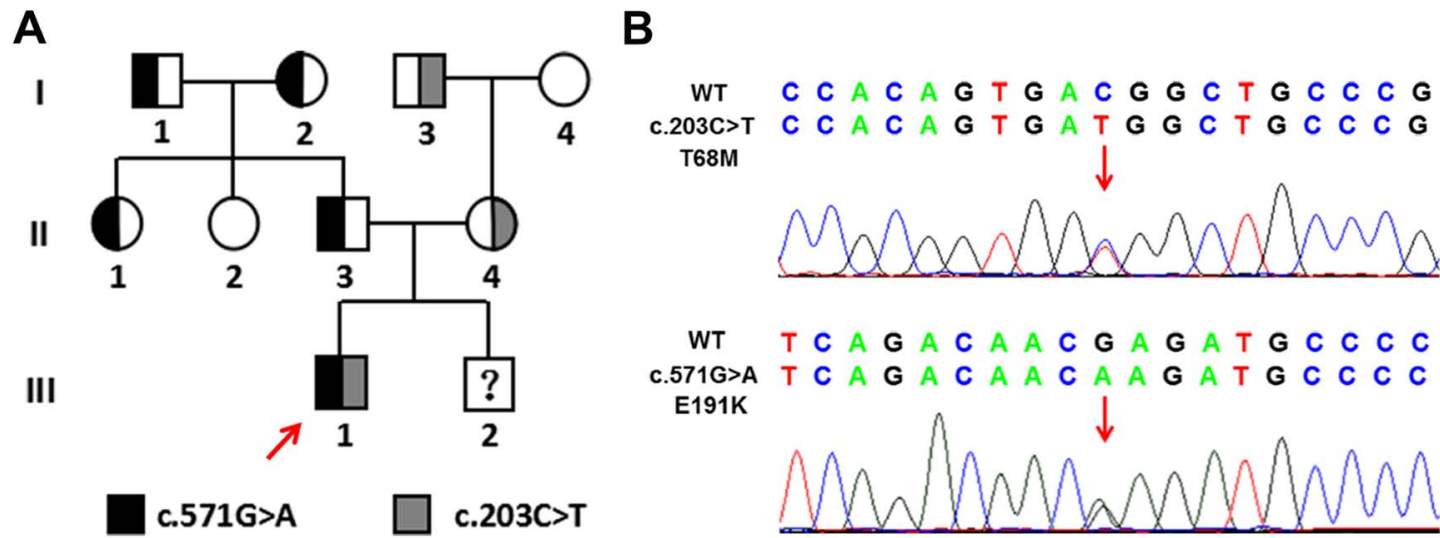

Figure 2. Family pedigree and mutation screening. (A) Family pedigree. The arrow indicates the proband. (B) DNA sequences of WT and mutant were validated using Sanger sequencing. Red arrows indicate mutations. WT, wild-type.

in ALPL of which the c.571G $>\mathrm{A}$ in exon 6 was from his father and the c.203C $>\mathrm{T}$ in exon 4 was from his mother.

Bioinformatics analysis was subsequently performed. Both variants are located in evolutionarily conserved regions, as indicated by UCSC Genome Browser (Fig. 3A). These variants were predicted to be harmful to humans according to PolyPhen-2 software and Mutation Taster analysis (data not shown). In addition, SIFT predicted that the c.203C $>$ T is likely to be harmful, while c. $571 \mathrm{G}>\mathrm{A}$ is tolerated (data not shown). SOPMA predicted that c. $203 \mathrm{C}>\mathrm{T}$ could result in alteration of the protein secondary structure, while c.571G $>$ A almost could not (data not shown). The three-dimensional structure of ALPL protein monomers predicted by I-TASSER showed a number of changes in the mutants (red arrows; Fig. 3C). The positions of these variants were marked by red (T68M) and purple $(\mathrm{E} 191 \mathrm{~K})$ in the structure of the protein dimer predicted by SWISS-MODEL, based on the homology to the placental isozyme (Fig. 3B). The E191K residue is located at the surface of the molecule and the T68M residue is located at the homodimer interface.

Immunofluorescence. Immunocytochemical staining of 293T cells transfected with anti-TNAP indicated the subcellular localization of the WT and mutated TNAP (Fig. 4). After transfection for $48 \mathrm{~h}$, the WT TNAP anchored to the surface of cells in general, which was shown by the clear fluorescence of cell membranes. Fluorescence in cells transfected with the ALPL-pE191K mutant was mainly located in the cell membrane. However, staining in the cytoplasm was increased relative to the WT. Almost no fluorescence was identified in 293T cells transfected with the ALPL-pT68M mutant construct.

Analysis of protein expression. Western blot analysis revealed identically sized bands of $\sim 80 \mathrm{kDa}$ in transfected cells expressing WT or mutant TNAP. However, the expression level of mutant ALPL was markedly lower compared with the WT, especially in cells transfected with the ALPL-pT68M mutant construct (Fig. 4).

Enzyme activity of ALP in WT and mutants. ALP activity in $293 \mathrm{~T}$ cells transfected with ALPL c. $203 \mathrm{C}>\mathrm{T}$ was $0.64 \%$ of WT ALPL enzyme activity after $24 \mathrm{~h}$, and $1.77 \%$ after $48 \mathrm{~h}$ (Fig. 4). In addition, the enzyme activity in $293 \mathrm{~T}$ cells transfected with ALPL c.571G $>$ A was $52.83 \%$ after $24 \mathrm{~h}$ and $72.05 \%$ after $48 \mathrm{~h}$. The results suggested that enzyme activity of p.T68M was almost completely lost and that of p.E191K was partially inactivated.

\section{Discussion}

HPP is an inborn error of metabolism characterized by low serum ALP activity (11). ALPL has been extensively studied as a pathogenic gene of HPP and many mutations in the ALPL gene are related to HPP (25). More than $75 \%$ 
A

HUMAN
CHIMP
RHESUS
MOUSE
RAT
DOG
OPOSSUM
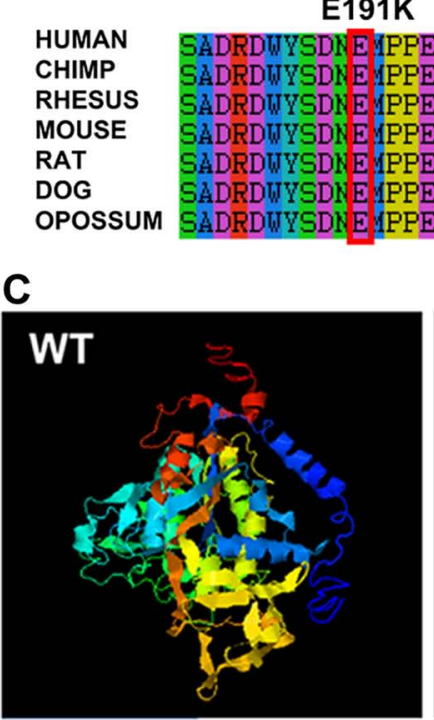

T68M

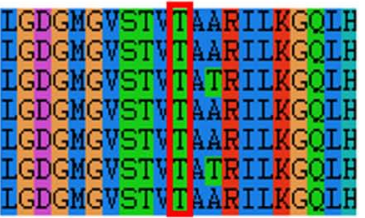

E191K

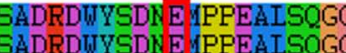

SADRDWYSDNE MPPEALSQGC

SADRDWYSDHE MPPEAISQGC

SADRDWYSDHE IPPEAISQGC

SADRDWYSDN E YPPEAISQGC

ADRDUYSDN F MPEALSOGC

SADRDWYSDNE MPPEAINQGC
B

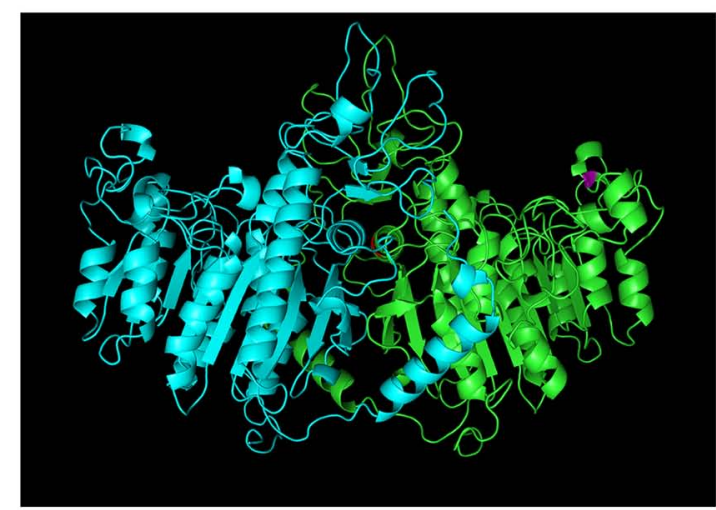

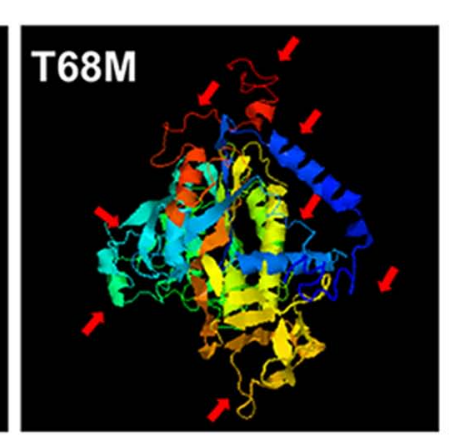

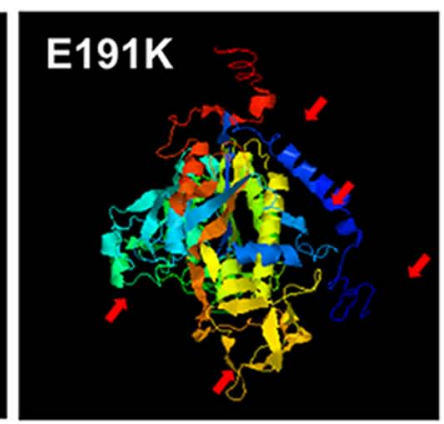

Figure 3. Bioinformatics analysis of the mutated protein. (A) Alignment of WT and mutant TNAP amino acid sequences. (B) 3D model of the protein dimer predicted by SWISS-MODEL. The position of the E191K residue is marked by purple and the T68M residue is marked by red. (C) Prediction of WT and mutant protein structures by I-TASSER. Red arrows denote the change of structure in the $\alpha$-helix or- $\beta$ sheet. TNAP, tissue-non-specific alkaline phosphatase; WT, wild-type.

are missense mutations and $>20 \%$ are splicing mutations, nonsense mutations, small deletions or insertions $(26,27)$. These loss-of-function mutations may affect the synthesis and trafficking of TNAP, and result in diminished expression and/or altered localization of TNAP (28-33). The present article described a child suffering from childhood HPP, with an onset at 1 year of age, who carried two heterozygous mutations c. 203C $>\mathrm{T}$ and c. $571 \mathrm{G}>\mathrm{A}$ in the ALPL gene. The serum TNAP activity of the proband's mother, who carried heterozygous c.203C $>\mathrm{T}$, was near the threshold, while that of his father, who carried heterozygous c. $571 \mathrm{G}>\mathrm{A}$, was normal. The c.571G $>$ A mutation is widely believed to be associated with childhood HPP.

The TNAP protein is synthesized as a $66 \mathrm{kDa}$ immature form; it is converted to an $80 \mathrm{kDa}$ mature form as it migrates from the endoplasmic reticulum to the Golgi apparatus and is finally fixed on the cell surface as a glycosylphosphatidylinositol-anchored protein $(33,34)$. The fluorescence intensity was increased in the cytoplasm of both mutants, which showed that partial protein was not anchored to the cytomembrane, and seemed to be more severe in T68M. A previous study suggested that transport of E191K (c.571G $>$ A) was delayed, and the fluorescence signal of the E191K protein was observed in the cytoplasm and cytomembrane after $24 \mathrm{~h}$ of transfection in COS-7 cells (31). The localization of E191K protein in $24 \mathrm{~h}$-transfected cells in the present study was consistent with this observation.

Western blotting demonstrated that the mature protein was decreased in E191K and T68M mutants compared with
WT. Levels of enzyme activity of mutant proteins expressed in $293 \mathrm{~T}$ cells also decreased compared with the WT. The T68M mutant indicated almost no serum activity after $24 \mathrm{~h}$ and $1.77 \%$ serum activity after $48 \mathrm{~h}$. A previous study also demonstrated very low enzyme activity $(\sim 4.5 \%)$ in the T68M mutant transiently expressed in COS-1 cells after $48 \mathrm{~h}$ (24). The results are consistent with the phenotype of family members in this pedigree. 3D model analysis suggested that the E191K residue is located at the surface of the molecule and may be associated with substrate approach and stabilization. The T68M residue is located at the homodimer interface and may serve an important role in maintaining the correct fold to allow homodimer interactions (35). A change in these positions may repress the process of post-translation and cause unsuccessful or delayed modification of the immature protein, which may then be degraded. This might be the reason for decreased enzyme activity in the mutants. According to previous reports, decreased TNAP activity can cause the accumulation of inorganic phosphate, which inhibits the formation of hydroxyapatite, thus preventing biomineralization $(16,19)$.

Herein, the current presented a molecular cytogenetic characterization of the compound heterozygous c. $571 \mathrm{G}>\mathrm{A}$ and c. 203C $>$ T mutations within ALPL that resulted in decreased protein expression and loss of enzyme activity with TNAP, leading to the childhood form of HPP in a six-year-old male patient. Potential genotype-phenotype correlations were discussed in the present case. The present data expanded the known ALPL compound heterozygous mutation spectrum 

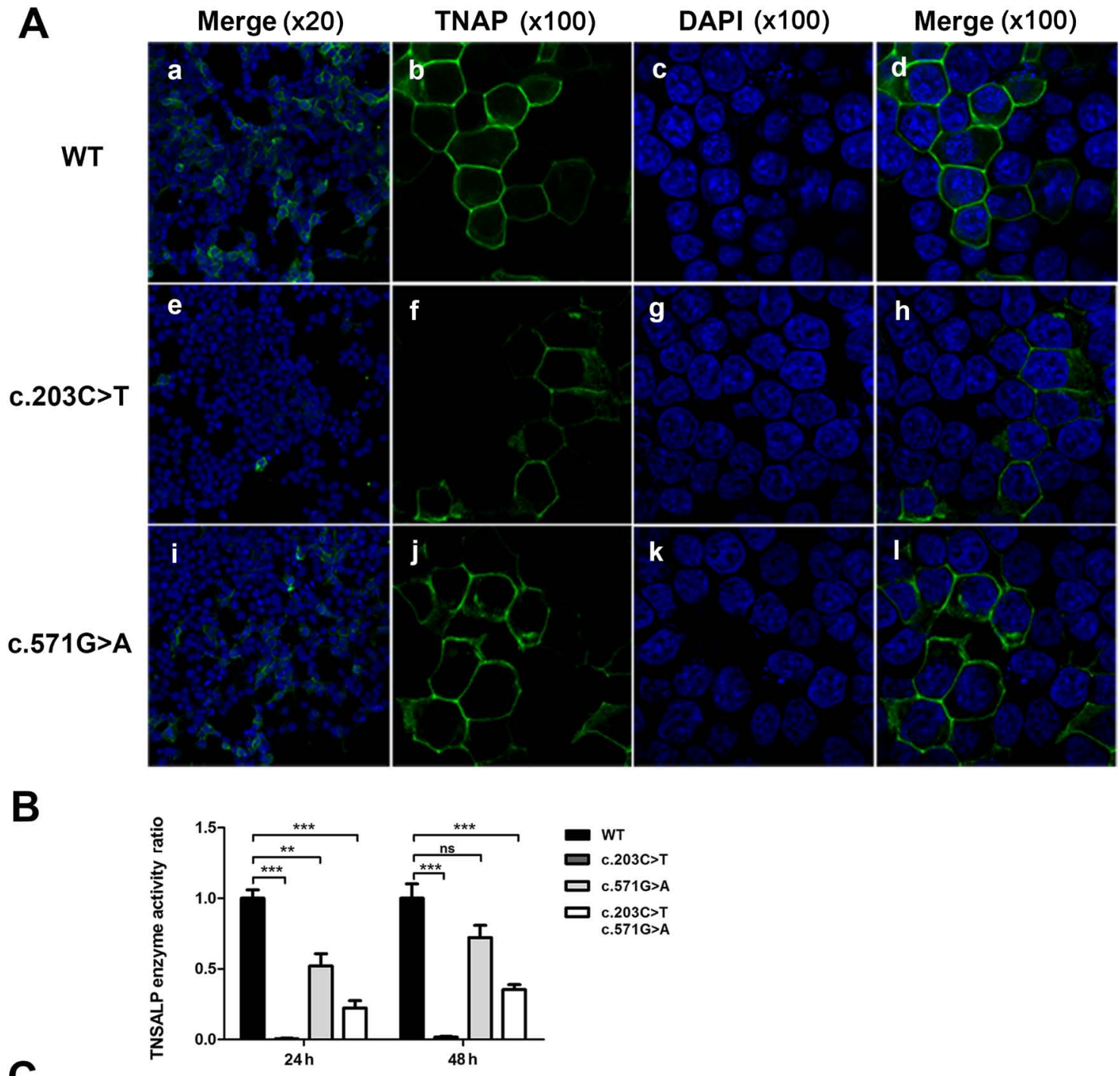

C
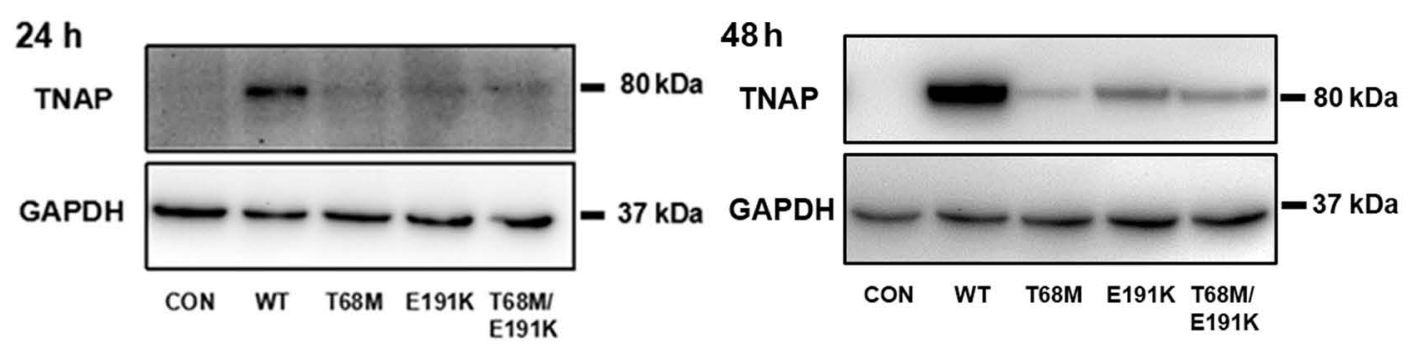

Figure 4. Functional study of WT and mutant TNAP. (A) Immunocytochemical staining of transiently transfected 293T cells indicated the subcellular localization of the WT, T68M and E191K. Green staining indicated TNAP and blue staining indicates the cell nucleus. (B) TNAP enzyme activity ratio between WT and mutants after transfections for 24 and $48 \mathrm{~h}$. (C) Expression levels of TNAP WT and mutants detected by western blotting. ${ }^{* *} \mathrm{P}<0.01$ and ${ }^{* * *} \mathrm{P}<0.001$. WT, wild-type; TNAP, tissue-non-specific alkaline phosphatase; CON, control.

and may potentially provide insight into the development of HPP.

\section{Acknowledgements}

Not applicable.

\section{Funding}

This study and publication costs were financially supported from the National Natural Science Foundation of China (grant no. 31970558), Natural Science Foundation of Guangdong Province (grant no. 2018B030311033), Science and Technology Planning Project of Guangdong (grant no. 2019A030317019) and Science and Technology Planning Project of Guangzhou (grant no. 201707010301).

\section{Availability of data and materials}

The datasets used and/or analyzed during the current study are available from the corresponding author on reasonable request. 


\section{Authors' contributions}

JW participated in clinical sampling and data collection. FX and XX designed the experiments. YL, XZ and XW performed genomic DNA isolation and mutation screening. $\mathrm{HH}, \mathrm{DG}$ and HS performed the functional studies. $\mathrm{HH}$ and FX wrote the manuscript. All authors read and approved the final manuscript.

\section{Ethics approval and consent to participate}

This study was approved by the Southern Medical University Ethics Committee. Written informed consent was obtained from the participants and the parents of the child.

\section{Patient consent for publication}

Written informed consent was obtained from the patient's legal guardian and his family for genetic analysis, publication of the research and the patient's images.

\section{Competing interests}

The authors declare that they have no competing interests.

\section{References}

1. Rathbun JC: Hypophosphatasia; a new developmental anomaly. Am J Dis Child 75: 822-831, 1948.

2. Orton NC, Innes AM, Chudley AE and Bech-Hansen NT: Unique disease heritage of the dutch-german mennonite population. Am J Med Genet A 146A: 1072-1087, 2008.

3. Greenberg CR, Evans JA, McKendry-Smith S, Redekopp S, Haworth JC, Mulivor R and Chodirker BN: Infantile hypophosphatasia: Localization within chromosome region 1p36.1-34 and prenatal diagnosis using linked DNA markers. Am J Hum Genet 46: 286-292, 1990.

4. Mornet E, Yvard A, Taillandier A, Fauvert D and Simon-Bouy B: A molecular-based estimation of the prevalence of hypophosphatasia in the European population. Ann Hum Genet 75: 439-445, 2011.

5. Mornet E: Hypophosphatasia. Orphanet J Rare Dis 2: 40, 2007.

6. Whyte MP: Genetics of Bone Biology and Skeletal Disease. Thakker RV, Whyte MP, Eisman J, Igarashi T (eds.). 1st edition. Academic Press pp337-360, 2012.

7. Whyte MP, Zhang F, Wenkert D, McAlister WH, Mack KE, Benigno MC, Coburn SP, Wagy S, Griffin DM, Ericson KL and Mumm S: Hypophosphatasia: Validation and expansion of the clinical nosology for children from 25 years experience with 173 pediatric patients. Bone 75: 229-239, 2015.

8. Mornet E and Nunes ME: Hypophosphatasia. In: GeneReviews ${ }^{\circledR}$. Adam MP, Ardinger HH, Pagon RA, et al (eds.). Seattle (WA): University of Washington, Seattle, 1993-2020.

9. Silver MM, Vilos GA and Milne KJ: Pulmonary hypoplasia in neonatal hypophosphatasia. Pediatr Pathol 8: 483-493, 1988.

10. Wenkert D, McAlister WH, Coburn SP, Zerega JA, Ryan LM, Ericson KL,Hersh JH, Mumm S and Whyte MP: Hypophosphatasia: Non-lethal disease despite skeletal presentation in utero (17 new cases and literature review). J Bone Miner Res 26: 2389-2398, 2011.

11. Fraser D: Hypophosphatasia. Am J Med 22: 730-746, 1957.

12. Whyte MP, Greenberg CR, Salman NJ, Bober MB, McAlister WH, Wenkert D, Van Sickle BJ, Simmons JH, Edgar TS, Bauer ML, et al Enzyme-replacement therapy in life-threatening hypophosphatasia. N Engl J Med 366: 904-913, 2012.

13. Whyte MP, Teitelbaum SL, Murphy WA, Bergfeld MA and Avioli LV: Adult hypophosphatasia: Clinical, laboratory, and genetic investigation of a large kindred with review of the literature. Medicine (Baltimore) 58: 329-347, 1979.

14. Sutton RAL, Mumm S, Coburn SP, Ericson KL and Whyte MP: Atypical femoral fractures' during bisphosphonate exposure in adult hypophosphatasia. J Bone Miner Res 27: 987-994, 2012.

15. Khandwala HM, Mumm S and Whyte MP: Low serum alkaline phosphatase activity and pathologic fracture: Case report and brief review of hypophosphatasia diagnosed in adulthood. Endocr Pract 12: 676-681, 2006.
16. Millan JL: The role of phosphatases in the initiation of skeletal mineralization. Calcif Tissue Int 93: 299-306, 2013.

17. Caswell AM, Whyte MP and Russell RG: Hypophosphatasia and the extracellular metabolism of inorganic pyrophosphate: Clinical and laboratory aspects. Crit Rev Clin Lab Sci 28: 175-232, 1991

18. Mornet E: Hypophosphatasia. Best Pract Res Clin Rheumatol 22: 113-127, 2008.

19. Whyte MP: Physiological role of alkaline phosphatase explored in hypophosphatasia. Ann N Y Acad Sci 1192: 190-200, 2010.

20. Saraff V, Narayanan VK, Lawson AJ, Shaw NJ, Preece MA and Högler W: A diagnostic algorithm for children with low alkaline phosphatase activities: Lessons learned from laboratory screening for hypophosphatasia. J Pediatr 172: 181-186.e1, 2016.

21. Cole DE: Hypophosphatasia update: Recent advances in diagnosis and treatment. Clin Genet 73: 232-235, 2008.

22. Combet C,Blanchet C, Geourjon C andDeléage G: NPS@: Network protein sequence analysis. Trends Biochem Sci 25: 147-150, 2000.

23. Henthorn PS, Raducha M, Fedde KN, Lafferty MA and Whyte MP: Different missense mutations at the tissue-nonspecific alkaline phosphatase gene locus in autosomal recessively inherited forms of mild and severe hypophosphatasia. Proc Natl Acad Sci USA 89: 9924-9928, 1992

24. Orimo H, Girschick HJ, Goseki-Sone M, Ito M, Oda K and Shimada T: Mutational analysis and functional correlation with phenotype in german patients with childhood-type hypophosphatasia. J Bone Miner Res 16: 2313-2319, 2001.

25. Weiss MJ, Cole DE, Ray K, Whyte MP, Lafferty MA, Mulivor RA and Harris H: A missense mutation in the human liver/bone/kidney alkaline phosphatase gene causing a lethal form of hypophosphatasia. Proc Natl Acad Sci USA 85: 7666-7669, 1988.

26. Mornet E, Hofmann C, Bloch-Zupan A, Girschick H and Merrer ML: Clinical utility gene card for: Hypophosphatasiaupdate 2013. Eur J Hum Genet 22: 2014.

27. Silvent J, Gasse B, Mornet E and Sire JY: Molecular evolution of the tissue-nonspecific alkaline phosphatase allows prediction and validation of missense mutations responsible for hypophosphatasia. J Biol Chem 289: 24168-24179, 2014.

28. Fukushi-Irie M, Ito M, Amaya Y, Amizuka N, Ozawa H, Omura S, Ikehara Y and Oda K: Possible interference between tissue-non-specific alkaline phosphatase with an Arg54->Cys substitution and acounterpart with an Asp277->Ala substitution found in a compound heterozygote associated with severe hypophosphatasia. Biochem J 348 (Pt 3): 633-642, 2000.

29. Ito M, Amizuka N, Ozawa $\mathrm{H}$ and Oda K: Retention at the cis-Golgi and delayed degradation of tissue-non-specific alkaline phosphatase with an Asn153->Asp substitution, a cause of perinatal hypophosphatasia. Biochem J 361(Pt 3): 473-480, 2002.

30. Ishida Y, Komaru K, Ito M, Amaya Y, Kohno S and Oda K: Tissue-nonspecific alkaline phosphatase with an $\operatorname{Asp}(289)->V a l$ mutation fails to reach the cell surface and undergoes proteasome-mediated degradation. J Biochem 134: 63-70, 2003.

31. Brun-Heath I, Lia-Baldini AS, Maillard S, Taillandier A, Utsch B, Nunes ME, Serre JL and Mornet E: Delayed transport of tissue-nonspecific alkaline phosphatase with missense mutations causing hypophosphatasia. Eur J Med Genet 50: 367-378, 2007.

32. Yang $\mathrm{H}$, Wang L, Geng J, Yu T, Yao RE, Shen Y, Yin L, Ying D, Huang R, Zhou Y, et al: Characterization of six missense mutations in the tissue-nonspecific alkaline phosphatase (TNSALP) gene in Chinese children with hypophosphatasia. Cell Physiol Biochem 32: 635-644, 2013.

33. Satou Y, Al-Shawafi HA, Sultana S, Makita S, Sohda M and Oda K: Disulfide bonds are critical for tissue-nonspecific alkaline phosphatase function revealed by analysis of mutant proteins bearing a C(201)-Y or C(489)-S substitution associated with severe hypophosphatasia. Biochim Biophys Acta 1822: 581-588, 2012.

34. Nasu M, Ito M, Ishida Y, Numa N, Komaru K, Nomura S and Oda K: Aberrant interchain disulfide bridge of tissue-nonspecific alkaline phosphatase with an Arg433->Cys substitution associated with severe hypophosphatasia. FEBS J 273: 5612-5624, 2006.

35. Mornet E, Stura E, Lia-Baldini AS, Stigbrand T, Ménez A and Le Du MH: Structural evidence for a functional role of human tissue nonspecific alkaline phosphatase in bone mineralization. J Biol Chem 276: 31171-31178, 2001.

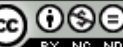

This work is licensed under a Creative Commons Attribution-NonCommercial-NoDerivatives 4.0 International (CC BY-NC-ND 4.0) License. 